\title{
What asteroseismology can teach us about low-mass core helium burning models
}

\author{
Thomas N. Constantino, Simon W. Campbell and John C. Lattanzio
}

Monash Centre for Astrophysics, School of Mathematical Sciencies

Monash University, Victoria 3800, Australia

email: thomas.constantino@monash.edu

\begin{abstract}
Standard models of low-mass core helium burning stars typically give an asymptotic $l=1$ g-mode period spacing well below that inferred from observed mixed modes. We find that most physical uncertainties, such as mixing beyond the fully convective core, are not significant enough to be responsible for such a discrepancy. The solution to the problem may lie in a deviation of structure away from its canonical form, such as a more massive H-exhausted core, which we briefly explore here.
\end{abstract}

Keywords. stars: horizontal-branch, stars: interiors, stars: late-type, stars: oscillations

\section{Introduction}

It has been posited for more than four decades that core helium burning ( $\mathrm{CHeB})$ stars develop a zone of slow mixing, or "semiconvection", beyond the fully convective core because of convective overshoot. The treatment of mixing in this region is a large source of uncertainty. Later evolution depends on the structure after this phase, and this is typically when the results of different stellar evolution codes begin to diverge.

Asteroseismology promises a unique insight into these stars and a chance to constrain our models of them. Bedding et al. (2011) have shown that it is possible to reliably distinguish $\mathrm{CHeB}$ stars from photometrically similar red giant branch (RGB) stars and Mosser et al. (2012) have developed a method to determine the gravity-dominated mixed mode period spacing $\Delta \Pi_{1}$ from the observed modes of mixed $\mathrm{p}$ and $\mathrm{g}$ character.

It has been found that our $\mathrm{CHeB}$ models from the Monash University stellar evolution code, irrespective of mixing scheme, have a range of asymptotic $\Delta \Pi_{1}$ below that inferred from observations of the Kepler red clump giants (Mosser et al. 2012). Similar results have been reported by Montalbán et al. (2013) using a different evolution code.

\section{Possible sources of this discrepancy}

The core mass of horizontal-branch stars has historically been constrained by matching stellar evolution isochrones to globular cluster colour-magnitude diagrams (e.g. Raffelt 1990). This method, however, depends on an interplay between RGB tip luminosity, initial helium abundance and a host of other factors (which are discussed in Catelan et al. 1996). If we assume that the red clump $\Delta \Pi_{1}$ determinations from Mosser et al. (2012) are representative, then we can more explicitly test our structure for zeroage horizontal-branch (ZAHB) models. ZAHB models should have a $\Delta \Pi_{1}$ that corresponds to the minimum observed since it then increases with age. This may in fact be the most direct probe of the mass of the H-exhausted core at the RGB tip. We have tested three different mixing schemes for the core: 1) using the Schwarzschild boundary with overshoot and 2) without overshoot, and 3) a new subroutine for semiconvection. 


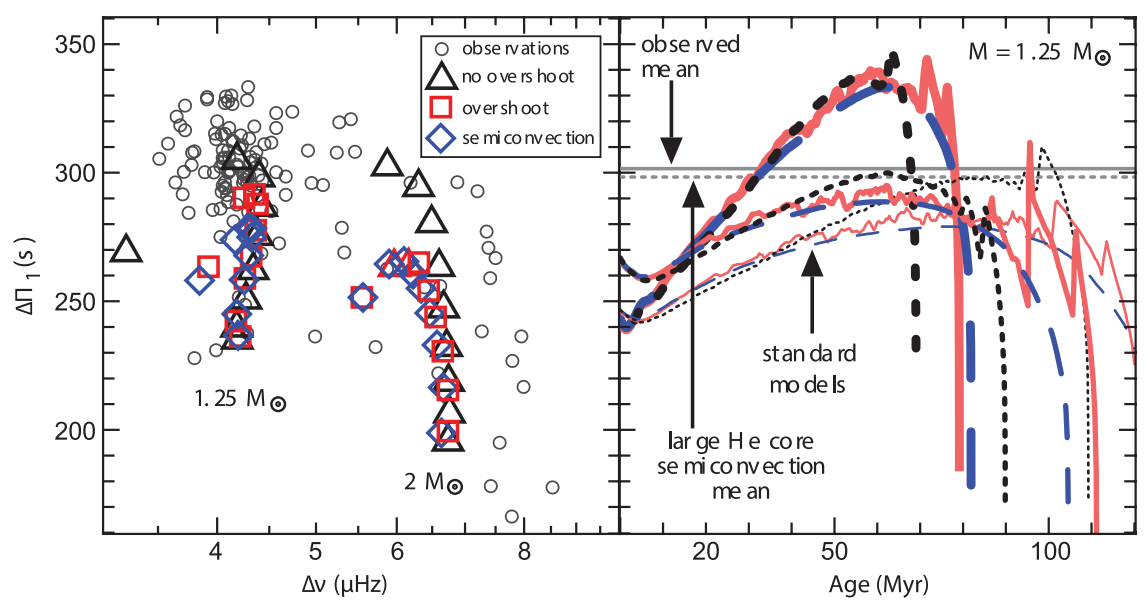

Figure 1. Left panel: $\Delta \Pi_{1}$ as a function of large frequency separation $\Delta \nu$ during the $\mathrm{CHeB}$ phase. Kepler observations are in circles (Mosser et al. 2012). Markers for $1.25 M_{\odot}$ and $2 M_{\odot}$ solar metallicity models are at 0.1 intervals in central helium mass fraction. No-overshooting, overshooting and semiconvection models are indicated by triangle, square and diamond markers respectively. Right panel: Evolution of $\Delta \Pi_{1}$ for standard red clump models (thin lines), models with a four-fold increase in neutrino emission (medium lines) and a case in which the H-exhausted core is artificially extended (thick lines). Models are $1.25 M_{\odot}$ and solar-metallicity. The solid straight line is the observed $\Delta \Pi_{1}$ mean when $\Delta \nu<5 \mu \mathrm{Hz}$. Short dashes, solid lines and long dashes correspond to mixing with no overshoot, overshoot and semiconvection respectively.

We find that none of these, however, can match the observations (Fig. 1). We also find that initial composition and microphysics such as reaction rates and equation of state are not significant factors.

Here we show that an experimental four-fold increase in neutrino emission on the RGB increases the core mass by $0.018 M_{\odot}$ at the flash, and in turn initially gives a $20 \mathrm{~s}$ increase in $\Delta \Pi_{1}$ (Fig. 1). This difference then diminishes over time because of slower H-burning and the consequently slower He core growth. In order to match the high end of the observed $\Delta \Pi_{1}$ range we need to artificially grow the He core so that by the end of $\mathrm{CHeB}$ it is around $0.1 M_{\odot}$ larger than in the normal case.

\section{Conclusions}

We have shown that uncertainties in mixing, reaction rates and composition do not appear to explain the discrepancy between the predicted g-mode period spacing from our models of red clump stars and that inferred from observations. We will conduct more detailed seismic analysis soon, with a view to determining whether or not there is indeed a shortcoming in our models and then uncovering the cause(s) of it. Other constraints such as cluster star counts will also be used.

\section{References}

Bedding, T. R., Mosser, B., Huber, D., et al. 2011, Nature, 471, 608

Catelan, M., de Freitas Pacheco, J. A., \& Horvath, J. E. 1996, ApJ, 461, 231

Montalbán, J., Miglio, A., Noels, A., Dupret, M.-A., Scuflaire, R., \& Ventura, P. 2013, ApJ, 766,118

Mosser, B., Goupil, M. J., Belkacem, K., et al. 2012, A\&A, 540, A143

Raffelt, G. G. 1990, ApJ, 365, 559 